\title{
Design of Color Recognition System Based on FPGA
}

\author{
Guibin Sun ${ }^{1}$, Zhi Weng ${ }^{1, *}$, Peng Zhao ${ }^{1}$, Dongxu Guo ${ }^{1}$, Yu Tian ${ }^{2}$ and Lei Xiao ${ }^{3}$ \\ ${ }^{1}$ College of Electronic Information Engineering, Inner Mongolia University, Hohhot 010021, China \\ ${ }^{2}$ College of Computer Science, Inner Mongolia University, Hohhot 010021, China \\ ${ }^{3}$ School of Mechatronic Engineering and Automation, Shanghai University, Shanghai 200072, China \\ ${ }^{*}$ Corresponding author
}

\begin{abstract}
Color recognition plays an important role in Machine Vision and Artificial Intelligence. In this article, a system based on FPGA applying to image acquisition, storage, display and color recognition is designed mainly to complete the recognition and calibration of fixed colors. Color images are captured by the analog camera in the system, which are decoded into standard YCbCr 4:2:2 data streams by AD chip. Then data streams are converted to the data of RGB video format by performing color space conversion. In the RGB color space, the color recognition method is proposed to identify the target color and calibrate on the display device. The experimental results demonstrate that the method can effectively achieve the identification of certain kinds of colors.
\end{abstract}

Keywords-FPGA; color recognition; color calibration; RGB

\section{INTRODUCTION}

Color recognition as a new detection technology has played an important role in Machine Vision and Artificial Intelligence. With the rapid development of science and technology, the research on color recognition is of great significance.

For color recognition of an object, the traditional method is to use a color sensor to measure the color components of the object in the RGB color space ${ }^{[1]}$. Then use the microprocessor to algorithm processing. Because test results are susceptible to environmental impacts and poor real-time, this detection method commonly used in close proximity detection and speed less demanding system. Now the more mature technology is using software methods to achieve color recognition in a PC (Personal Computer) as a representative of a serial processor. The ways mainly collect images through image acquisition card, and complete color recognition and other processing in the PC, then sending the results to the implementing agencies to execute. Obviously this implementation is of poor real-time, a higher power consumption and not easy to carry. With the development of semiconductor technology and digital signal processing technology, digital signal processing ASIC has been rapidly developed. The most representative of which is the generation of DSP (Digital Signal Processor) ${ }^{[2]}$, it has changed drawbacks that the PC performs image processing and made the digital signal into embedded era. However, DSP has also been many restrictions in the acquisition and processing of high-definition images due to its speed problems of serial processing in it.

To solve the above problem, the article designs a system which bases on FPGA applying to image acquisition, storage, display and color recognition and presents a color recognition method to complete the recognition and calibration of fixed colors for color images. FPGA is a large-scale programmable logic devices, it has a unique advantage in real-time digital signal processing. The processing speed of FPGA is much faster than the PC and DSP because which program statements are executed in parallel.

\section{SyStEM Module DESIGN}

\section{A. System Design Diagram}

The system mainly includes four parts: image acquisition module, data caching module, the FPGA core module and VGA display module. Block diagram of overall system design is shown in Figure 1:

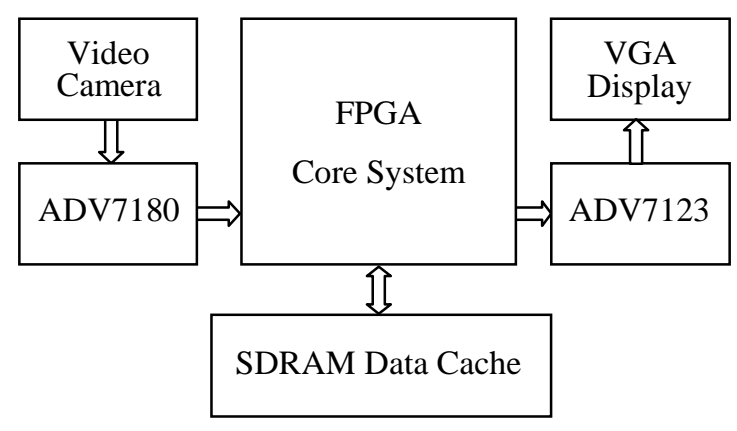

FIGURE I. SYSTEM STRUCTURE DIAGRAM

\section{B. Image Acquisition}

In this design, the analog camera from Dahua Company is used as video input, which can display more clearly than ordinary CMOS digital camera. The chip which converts analog signal into digital signal in collection process is ADV7180. The chip is a video decoder chip launched from ADI Company that can capture analog signal in PAL and NTSC formats. After the adoption of the IIC bus is configured correctly, the chip can automatically detect the input format, and convert it to component video image data in standard YCbCr 4:2:2 ${ }^{[3]}$. Then the data is passed to SDRAM through the FPGA.

\section{Data Cache}

For doing data caching within the FPGA, they would occupied a large number of logic cells if the internal logic 
constitute the memory, so Altera Company integrates many M9K memory within the FPGA. However, it is not enough for a large number of the image data cache to only use the internal memory apparently. Therefore, in this design, a 32 Mx16 bit of SDRAM is used to constitute the data buffer module, storing the data stream which is decoded by the ADV7180. The FPGA reads the data from SDRAM and converts it into YCbCr 4:4:4 data formats.

\section{FPGA Core System}

This design uses EPCE115F29C7 as the core processor, which is FPGA from the Cyclone IV series of Altera Company. The FPGA has rich resources to meet the design requirements for real-time color recognition and calibration of objects.

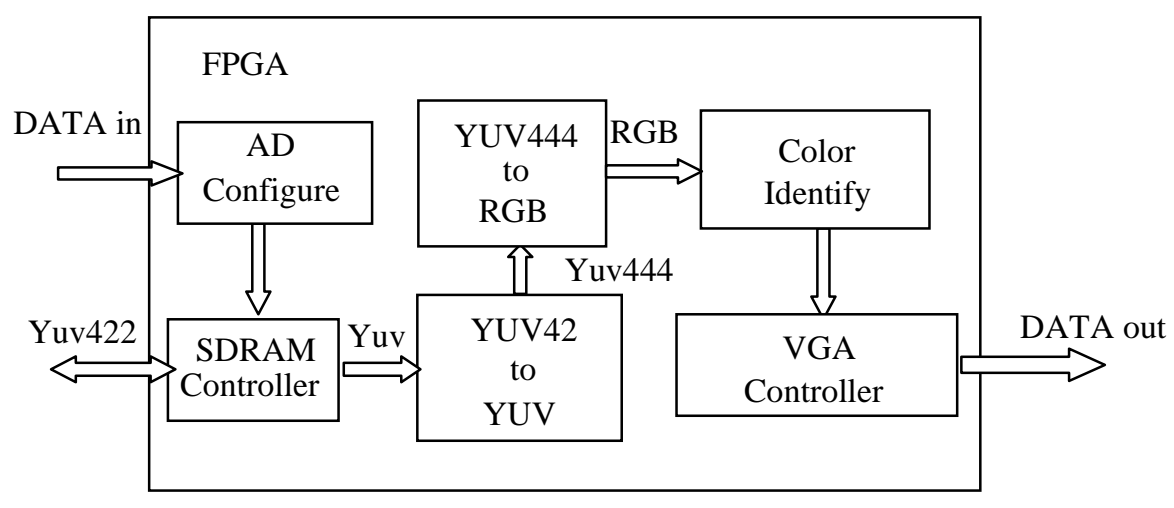

FIGURE II. FPGA INTERNAL MODULE DESIGN

Figure 2 is the functional modules design within the FPGA. ADConfigure module configures the decoder chip ADV7180 through the IIC bus, and then receives the YUV422 digital signals from decoder chip. Module SDRAM Controller makes timing control to external memory SDRAM and stores the YUV 4:2:2 digital signals into the SDRAM. The function of which the module of YUV422toYUV444 and YUV444toRGB is respectively converts YCbCr 4:2:2 signals to YCbCr 4:4:4 and the YCbCr 4:4:4 signals to RGB signals. Color recognition and calibration of objects is done in Color Identify module through processing each color component of the RGB signal to achieve color recognition. Finally, the module Color Identify transmits RGB signals processed by the module VGA Controller. The module VGA Controller transmits the RGB signals to digital-analog converter chip ADV7123 in order to achieve VGA display.

\section{E. VGA Display}

The design uses a high-speed digital-analog converter chip ADV7123 to convert the RGB digital signal to standard VGA video transmission signals to complete the $640 \times 480$ image display.

\section{COLOR RECOGNITION PRINCIPLES}

\section{A. Color Space Theory}

This design mainly uses the YUV and RGB color model.

YUV is a color encoding method used by European television system. Wherein "Y" represents brightness, and "U" and " $\mathrm{V}$ " indicates chrominance, which is the hue and saturation of the image. YUV mostly refers to YCbCr under normal circumstances. " $\mathrm{U}$ " is equivalent to "Cb" and " $\mathrm{V}$ " is equivalent to "Cr" in this article.
RGB color space commonly used in image display, which represent various colors by mixed weighted of three primary colors red, green and blue. It is shown in Figure 3 unit cube. Three-axis of three-dimensional space respectively corresponds to red, green and blue components. The origin indicates black and the farthest point represents white ${ }^{[4]}$. Every point inside the cube or surface corresponds to different colors.

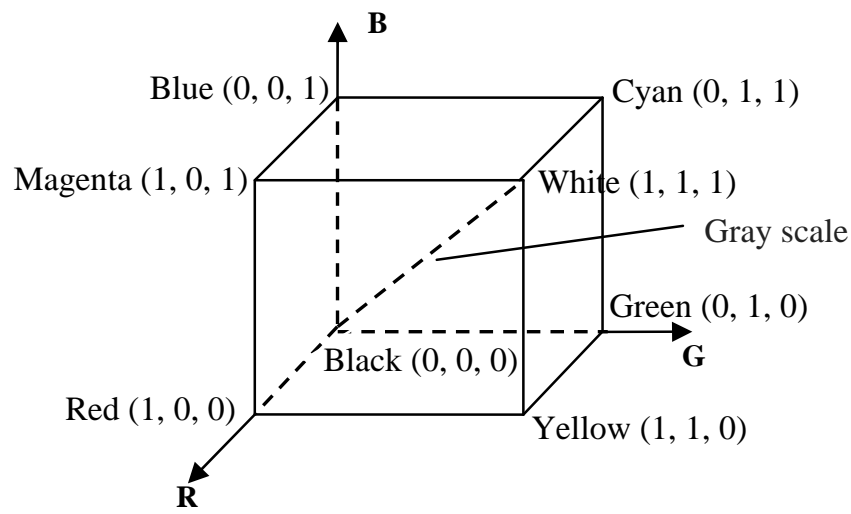

FIGURE III. RGB COLOR SPACE

\section{B. Color Space Conversion}

In this design, the output of image acquisition module is YCbCr data stream format. But RGB data format is used in color recognition and image display. So it is necessary to convert the YCbCr color model to RGB color model. The conversion formula is as follows ${ }^{[5]}$. 


$$
\left[\begin{array}{l}
R \\
G \\
B
\end{array}\right]_{=}\left[\begin{array}{ccc}
1 & 0 & 1.402 \\
1 & -0.34414 & -0.71414 \\
1 & 1.772 & 0
\end{array}\right]\left[\begin{array}{c}
Y \\
C b \\
C r
\end{array}\right]_{+}\left[\begin{array}{c}
-179.45 \\
135.459 \\
-226.816
\end{array}\right]
$$

In the FPGA, you cannot directly operate on floating-point operations, so firstly amplify several times about the equation around for computing. Then narrow the same multiples after the operation finished. The experimental verifies that the result is better while amplifying the 596 times. Results enlarged as follows ${ }^{[6]}$ :

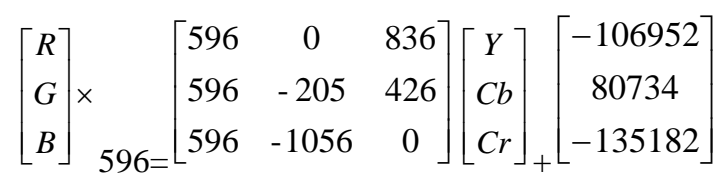

Since ADV7123 is 10-bit digital signal input, so using 10-bit digital signal respectively represent $R, G$, and $B$ component values. Because RGB values may be outside the domain after the transform, do limiting process to RGB value after converting. If RGB value is greater than 1023, then give it a value of 1023 . If less than 0,0 is assigned.

\section{Color Recognition}

This article gives a way of color identification whose advantage lies in removing the main component of the pixel brightness value. Because the brightness decides the body's shadow, while the existence of shadow makes the same color changed significantly, leading to the wrong color identification. By removing the brightness component, the target color can be detected effectively by a single detection algorithm.

$$
\left[\begin{array}{c}
Y \\
C b \\
C r
\end{array}\right]_{=}\left[\begin{array}{ccc}
0.299 & 0.587 & 0.114 \\
-0.687 & -0.3313 & 0.500 \\
0.500 & -0.1487 & -0.0813
\end{array}\right]\left[\begin{array}{l}
R \\
G \\
B
\end{array}\right]_{+}\left[\begin{array}{c}
0 \\
128 \\
128
\end{array}\right]
$$

The formula (3) is a conversion from the model RGB to the model YCbCr, which can be expressed as the brightness component:

$$
\mathrm{Y}=0.299 \mathrm{R}+0.587 \mathrm{G}+0.114 \mathrm{~B}
$$

From formula (4) we can infer that, the green component takes up $58.7 \%$ proportion, which means the body's brightness decided by green component. Therefore, in the process of color identification, the green component can be removed. Meanwhile, the main component of the pixel brightness value can also be removed, from which we can identify the body's color effectively.

From the above conclusion, assume two variables "m" and "n", which contains the following relationship:
$\mathrm{m}=\mathrm{R}-\mathrm{G}$

$$
\mathrm{n}=\mathrm{B}-\mathrm{G}
$$

For the target color, it corresponds a unique "m" and "n". Just by giving the limitation of " $m$ " and " $n$ ", the target color can be identified successfully. After the verification of experiments, the accuracy rate can achieve more than $87 \%$.

\section{EXPERIMENTAL RESULTS AND ANALYSIS}

The hardware platform used in the design system is DE2-115 Development Board from Terasic Company. The camera used is infrared analog camera from Dahua Company.

Figure 4 for the color recognition system hardware connection diagram. The system uses the wall containing different colors to make a color recognition test. When the system detects a target color, use a red box of $16 \times 16$ to calibrate target area. In order to avoid the background color except the color wall to impact the test results in programming, setting the target column color recognition range is $112 \sim 528$, row identifying the range of 96 to 384. As shown below, the red color recognition rendering is shown in Figure 5.

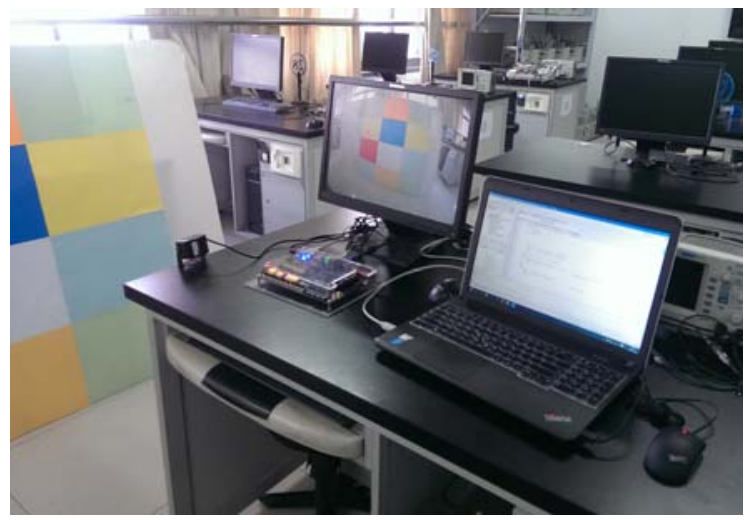

FIGURE IV. SYSTEM HARDWARE CONNECTION DIAGRAM

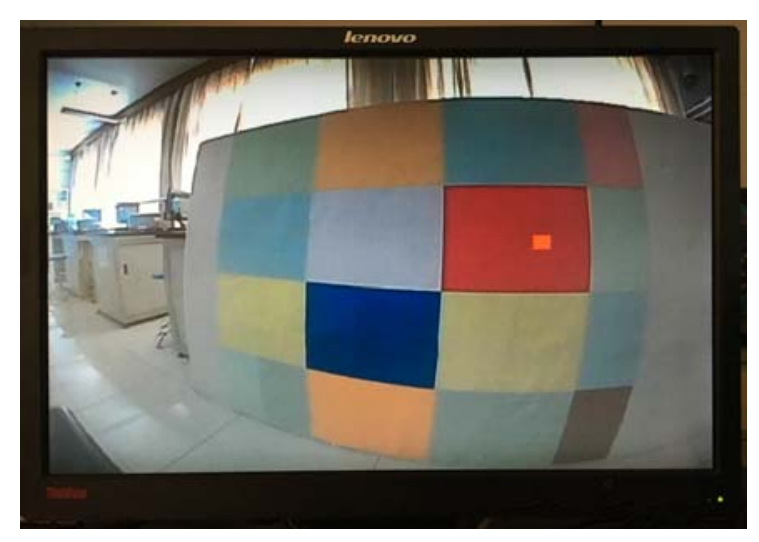

FIGURE V. RED COLOR RECOGNITION 


\section{CONCLUSION}

The article designs a system which bases on FPGA applying to image acquisition, storage, display and color recognition. The system has solved the problem that color sensor to identify the color affected by light and environment change. Simultaneously, the speed of the system is superior to DSP in the image acquisition, storage and processing. Through experimental verification, the identification of color accuracy is higher and the recognition algorithm is simple, convenient and practical in this design system. It can be used to identify color in the field of Machine Vision and Artificial Intelligence.

\section{ACKNOWLEDGEMENT}

This research was financially supported by National College Students Innovation and Entrepreneurship Training Program under Grant 201510126032.

\section{REFERENCES}

[1] Tang Sheng Xue, Liu Bo Feng, Xu Dong Feng, Color recognition method based on fuzzy neural network, Journal of Transducer Technology, 22(11):57-59,2003.

[2] Yao Wang, Jorn Ostermann, Ya Qing Zhang, Video Processing and Communications, Publishing House of Electronics Industry, Beijing, China, 2003.

[3] Lin Zhi Qiang, Meng Ling Jun, Peng Qing Qing, Design of Real-time Image Acquisition Systerm Based on ADV7180, Video Engineering, 17(35):36-39, 2011.

[4] Huang Zhi Hui, Li Rong, Design and implementation of color recognition touch-screen system based on FPGA, Modern Electronics Technique, 38(10):61-64,2014.

[5] Liu He, Digital Image Processing and Applications, China Electric Power Press, Beijing, China, 2006.

[6] Liu Qing Tang, Wang Zhong Hua, Chen Di, Introduction to Digital Media Technology, Tsinghua University Press, Beijing, China, 2008. 